\title{
Coxiella burnetii Detected in Tick Samples from Pastoral Communities in Kenya
}

\author{
Hellen Koka (D), ${ }^{1,2}$ Rosemary Sang, ${ }^{3,4}$ Helen Lydia Kutima, ${ }^{1}$ and Lillian Musila ${ }^{2}$ \\ ${ }^{1}$ Jomo Kenyatta University of Agriculture and Technology, P.O. Box 62000-00200, Nairobi, Kenya \\ ${ }^{2}$ US Army Medical Research Directorate-Kenya, P.O. Box 606-00621, Nairobi, Kenya \\ ${ }^{3}$ Kenya Medical Research Institute, Centre for Virus Research, P.O. Box 54628-00200, Nairobi, Kenya \\ ${ }^{4}$ International Centre for Insect Physiology and Ecology, P.O. Box 30772-00100, Kenya \\ Correspondence should be addressed to Hellen Koka; hellen.koka@usamru-k.org
}

Received 28 February 2018; Revised 23 April 2018; Accepted 5 June 2018; Published 9 July 2018

Academic Editor: Maria Ogrzewalska

Copyright (c) 2018 Hellen Koka et al. This is an open access article distributed under the Creative Commons Attribution License, which permits unrestricted use, distribution, and reproduction in any medium, provided the original work is properly cited.

\begin{abstract}
Ticks are important disease vectors in Kenya with documented evidence of carriage of zoonotic pathogens. Coxiella burnetii is an important tick-borne pathogen that is underreported in Kenya and yet this infection likely contributes to undiagnosed febrile disease in pastoral communities. Archived human blood (278) and tick pool samples (380) collected from five pastoral communities in Kenya were screened for C. burnetii by PCR using primers targeting the transposon-like IS1111 region. All the human blood samples were negative for C. burnetii DNA. However, C. burnetii was detected in 5.53\% (21/380) of the tick pools tested. Four of the twenty-one PCR positive samples were sequenced. The findings indicate that Coxiella burnetii was not present in the human blood samples tested. However, C. burnetii was detected in ticks from Mai Mahiu, Marigat, Ijara, Isiolo, and Garissa indicating a natural infection present in the tick vector that poses a risk to livestock and humans in these communities.
\end{abstract}

\section{Introduction}

Q fever, a zoonotic disease caused by Coxiella burnetii, has emerged as an important human and veterinary public health problem worldwide [1]. In humans, Q fever may be asymptomatic or manifest as an acute febrile illness with pneumonia [2]. In a small number of patients, the disease may progress to a chronic form that is mainly associated with patients who are immunocompromised [3]. It may also be seen in patients who have preexisting heart valve defects presenting as endocarditis $[4,5]$.

Domestic animals such as cattle, sheep, and goats are the primary reservoirs of C. burnetii [6]. In animals, infections are mainly asymptomatic but still births, late abortion, delivery of weak offspring, and infertility are reported to occur [7]. The infected animal sheds the bacterium through the placenta and birth fluids which may contaminate the environment. Contamination of the environment leads to airborne dissemination of the bacterium and infection of persons in close contact with livestock [8-10]. As a result, $\mathrm{Q}$ fever is often an occupational disease affecting farmers, veterinarians, and abattoir workers [5].
Although transmission to humans and susceptible animals occurs primarily through inhalation of C. burnetii spores from the environment, humans may also get infected through contact with milk, urine, faeces, vaginal mucus, or semen of infected animals [11]. Cases of Q fever in man are mainly precipitated by infection in animals [12] yet, an outbreak of Q fever in humans is what leads to the investigation of livestock in most countries [9]. In fact, an outbreak in the human population can be prevented by monitoring domestic animals for signs of abortion or birthing of weak offspring which indicate possibility of $\mathrm{Q}$ fever infection [13].

Ticks are considered the natural primary vector of $C$. burnetii as they maintain the infection in domestic animals [5]. Transmission of C. burnetii is by tick bite or exposure to infected excreta expelled by ticks onto the skin of the animal host as they feed $[4,7]$. In experimental studies, ticks readily transmit $C$. burnetii to humans [14]. However, direct transmission to humans from an infected tick is not well documented and may occur only rarely in nature [15]. The main transmission route from ticks to humans is therefore 
considered to be via inhalation of contaminated fecal material from ticks $[1,4]$.

Outbreaks of $\mathrm{Q}$ fever in man and domestic animals have been reported in many European countries [1, 16, 17] but since $\mathrm{Q}$ fever is not routinely tested for, it is likely underreported in Africa [12]. Since there is a growing interest in the role of nonmalarial causes of fevers [2], documented reports of $\mathrm{Q}$ fever infections are increasing as more diagnostic tools become available [18]. For instance, a study in Tanzania demonstrated that Q fever was the cause of 5\% of febrile illness in hospitalized adult and paediatric patients [19]. Another study in Namibia, evaluating the causes of febrile illness, reported a $26 \%$ seroprevalence of Q fever in blood donors [20]. Furthermore, a study in Chad evaluating zoonotic diseases in three nomadic communities reported a $1 \%$ prevalence rate of Q fever [21].

In Kenya, the prevalence of Q fever was reported at $12.1 \%$ in both livestock and human populations in five of seven provinces [22]. The North Eastern and upper Eastern regions of Kenya which have large nomadic pastoral communities were not included in the sero-survey, yet they keep large herds of livestock [23] and the risk of Q fever transmission is reportedly higher in grazed animals [11]. Although it is clear that livestock husbandry systems play a key role in the transmission of $Q$ fever to humans, infection rates in nomadic communities in Kenya have not been determined. The fact that Q fever is reportedly higher in grazed animals and that nomadic pastoral communities keep large herds of livestock, a study to determine the prevalence of Q fever (C. burnetii) in human and tick samples from several pastoral communities in Kenya was carried out.

\section{Materials and Methods}

2.1. Ethical Approval. This study was approved by the Institutional Review Boards at Kenya Medical Research Institute (KEMRI study \# 2454) and the Walter Reed Army Institute of Research (WRAIR study \#2099). Collection of human samples and ticks had been previously approved by the KEMRI IRB (Study \#1560 and \#824) and WRAIR IRB study \#1134. The study set out to test for Rickettsia spp., Babesia spp., and Coxiella burnetii in human samples and ticks from five pastoral communities (Figure 1) and a thesis was generated [24]. The results on Rickettsia spp. detected from this study were reported recently [25]. Herein, we present the results on detection of Coxiella burnetii.

2.2. Human Blood and Tick Samples. The human samples had been collected between December 2011 and December 2012 at dispensaries in three sites: Marigat, Mai Mahiu, and Ijara. Whole blood samples were collected from children $>1$ year of age and adults presenting at dispensaries with unexplained fever $\left(>37.5^{\circ} \mathrm{C}\right)$ and other symptoms including diarrhoea, chills, muscle aches, joint pains, coughs, abdominal pain, and vomiting. Demographic data that had been collected from the study subjects included date of collection, village of residence, age in years, sex, occupation, tick bite, symptoms, clinician's diagnosis, and contact with animals.

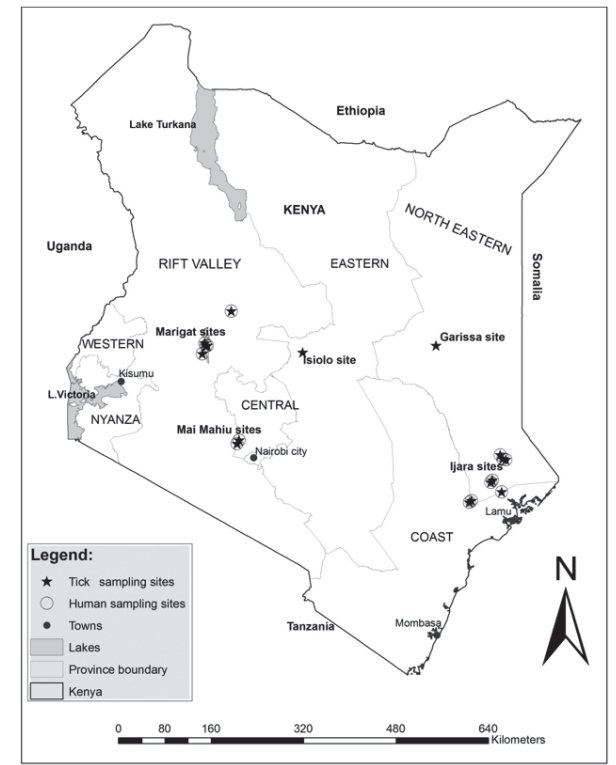

FIGURE 1: Map of sites where ticks and human samples had been collected.

The 380 tick pools tested in this study were collected from Marigat, Mai Mahiu, Ijara, Garissa, and Isiolo. Whole adult ticks had been identified to the species level using two tick identification keys. The ticks were then pooled in groups of 1 to 8 according to sex, developmental stage, species, area, site, collection date, and host. The tick pools were identified, homogenized, and processed as described by Koka et al., 2017 [25].

2.3. DNA Extraction and Polymerase Chain Reaction Amplification. DNA was extracted from both tick and human samples using the Qiagen DNeasy Blood and Tissue kit (Qiagen Inc., Valencia, CA). The DNA was quantified using a Nanodrop 2000 spectrophotometer (Thermo Fisher scientific) and stored at -70 to $-80^{\circ} \mathrm{C}$. Coxiella burnetii was detected using a single step conventional PCR assay using the primers Trans 1 and Trans 2 [4] designed to amplify a 687-bp fragment of the repetitive, transposon-like IS1111 region. The PCR amplification conditions for the Trans primers included an initial denaturation step at $95^{\circ} \mathrm{C}$ for $2 \mathrm{~min}$, followed by five cycles at $94^{\circ} \mathrm{C}$ for $30 \mathrm{~s}, 66$ to $61^{\circ} \mathrm{C}$ (the temperature was decreased by $1^{\circ} \mathrm{C}$ between consecutive steps) for $1 \mathrm{~min}$, and $72^{\circ} \mathrm{C}$ for $1 \mathrm{~min}$. These cycles were followed by 35 cycles of $94^{\circ} \mathrm{C}$ for $30 \mathrm{~s}, 61^{\circ} \mathrm{C}$ for $30 \mathrm{~s}$, and $72^{\circ} \mathrm{C}$ for $1 \mathrm{~min}$ and then a final extension step of $10 \mathrm{~min}$ at $72^{\circ} \mathrm{C}$. Coxiella burnetii DNA was used as a positive control and water was used as a negative control. The PCR assays were performed in a Gene Amp 9700 thermocycler (Applied Biosystems) using a Taq PCR master mix kit (Qiagen Inc., Valencia, CA), 1ng of template DNA, and $1 \mu \mathrm{l}$ of $50 \mu \mathrm{M}$ of the Trans primer in a $25 \mu \mathrm{l}$ reaction mix. PCR products were separated on a $2 \%$ agarose gel visualized with ethidium bromide on a UV transilluminator. Products were sized using an O'rangeRuler 100bp DNA ladder (Thermo Fisher Scientific). 
2.4. Sequencing and Data Analysis. Positive PCR products were purified and sequenced. The nucleotide sequences obtained in this study are available in GenBank under accession numbers: MG710507-MG710510. Sequencing and statistical analysis of the data were carried out as previously described by Koka et al. 2017 [25].

\section{Results}

3.1. Prevalence of Coxiella burnetii in Ticks. All the human blood samples were negative for C. burnetii DNA. On the contrary, C. burnetii was detected in 5.53\% (95\% CI 3.458.32) of the tick pools tested. The prevalence of C. burnetii varied significantly $(\mathrm{p}=0.006)$ across the sites with Mai Mahiu recording the highest prevalence at $13.16 \%$, followed by Marigat (7.89\%), Isiolo and Ijara (both 2.63\%), and Garissa recording only a single positive tick pool. The number of tick pools positive for $C$. burnetii was not significant with respect to animal host $(\mathrm{p}=0.152)$ : sheep $(10.61 \%)$, goat $(5.34 \%)$, and cattle $(4.58 \%)$, and no tick pools from camels were positive. C. burnetii (Table 1) was predominantly detected in Rhipicephalus tick species (95.2\%).

3.2. Coxiella burnetii Identified from the PCR Positive Tick Samples. Four of the twenty-one C. burnetii positive samples from ticks were sequenced and compared with sequences in GenBank. Two of the amplicons sequenced were derived from Rh. evertsi evertsi tick pools from Mai Mahiu, one from sheep and one from cattle. The other two amplicons were from $R h$. pulchellus tick pools from Garissa and Isiolo, collected from a goat and cattle, respectively. All four $C$. burnetii positive tick samples were 94-97\% homologous to the virulent strain $\mathrm{CbCVICl}$ and strain Heizberg from the Netherland outbreak.

\section{Discussion}

4.1. Prevalence of Coxiella burnetii in Tick Samples. C. burnetii was detected in a small percentage of ticks (5.5\%) collected from sheep, goats, and cattle. The prevalence rate is comparable to the $6.4 \%$ reported in ticks collected from cattle in Ethiopia [26]. On the contrary, a study done in rural Western Kenya reported a lower prevalence of $2.5 \%$ in ticks collected from cattle [27] where zero grazing is commonly done. The slightly higher prevalence of C. burnetii in ticks in this study may be associated with the livestock rearing practice of grazing animals in the pastoral communities [11].

40 species of ticks are known to be infected with C. burnetii worldwide [1]. Rh. evertsi evertsi, the most widespread species of all the Rhipicephalus ticks in Africa (Walker 2003), was the predominant tick species infected with C. burnetii in this study. This is consistent with other studies in Senegal, Nigeria, and Kenya which detected C. burnetii DNA in $R h$. evertsi evertsi tick species [18, 28, 29]. The detection of $C$. burnetii DNA in Rh. appendiculatus in this study corroborates previous reports of the same findings in Kenya [18, 27]. In addition, C. burnetii DNA was detected in $R h$. pulchellus and A. gemma tick species. C. burnetii infection in these two tick species has been reported before in Ethiopia [26] while $C$. burnetii infection in $R h$. pulchellus was recently reported in Kenya [18].

The ticks in our study were collected from sheep, goats, and cattle but these ticks also feed on donkeys, horses, and wild ungulates [30]. Ticks are able to transmit C. burnetii to these animals when they take a blood meal but some ticks are also able to transmit the infection transovarially [28]. We postulate that the ticks in our study may be involved in the transmission cycle of $C$. burnetii between domestic animals and wildlife. This is corroborated by the detection of C. burnetii from $R h$. evertsi evertsi, $R h$. appendiculatus, and $R h$. pulchellus ticks collected from zebras and buffalos in Laikipia [18]. Importantly, C. burnetii DNA was not detected in ticks collected from camels in our study, despite a high C. burnetii seroprevalence being reported in camels in the region $[31,32]$.

The study also indicated a distinct geographical distribution of the positive ticks, with ticks from Mai Mahiu and Marigat being significantly more infected with $C$. burnetii than the other sites. Furthermore, the C. burnetii positive tick samples that were sequenced were closely related to the virulent strain $\mathrm{CbCVIC1}$ and strain Heizberg associated with the largest outbreak of C. burnetii in the Netherland in 20072010 [33]. The sequenced samples were also related to the virulent Cb 175 epidemic strain from French Guyana known to cause the highest prevalence of community acquired pneumonia in the world [12]. Therefore, the animal and human populations in these two sites are at considerably higher risk of getting a $Q$ fever infection. An outbreak of $\mathrm{Q}$ fever in humans had been reported in Baringo, by the Zoonotic Disease Unit in Kenya in March 2014 [34]. However, in this study C. burnetii DNA, the causative agent of Q fever, was not detected in the human blood samples tested. This is in spite of activities such as herding, slaughtering of cattle, and milking that allow contact with infected animals which are carried out in these communities [35].

A limitation of this study was the lack of human samples from Garissa and Isiolo which prevented direct correlation of human and tick infections. No human samples were collected from these sites in the previous studies from which the archived samples were obtained. Another limitation of this study was the time difference in sampling humans and ticks in these sites making it challenging to infer tick to human transmission. A distinct seasonality of $\mathrm{Q}$ fever related to the parturient season in domestic and wild animal has been established [36]. Therefore, it is likely that samples collected in the parturient season would provide different results.

\section{Conclusion}

The study identified $R h$. evertsi evertsi and Rh. pulchellus as the tick species predominantly infected with $C$. burnetii. The findings in this study suggest that grazing of cattle, sheep, and goats in the pastoral communities increases the risk of acquiring $C$. burnetii infection from ticks. We suggest that a robust surveillance system in domestic animals and humans be established to monitor this disease in these two locations. More emphasis should also be placed on acaricide treatment of all livestock to prevent the spread of tick-borne infection. 
TABLE 1: Tick species collected from livestock and the tick species positive for Coxiella burnetii.

\begin{tabular}{|c|c|c|c|c|c|c|c|}
\hline \multirow[t]{2}{*}{ Tick species } & \multicolumn{4}{|c|}{ \# ticks per animal host } & \multicolumn{3}{|c|}{ Coxiella PCR positive pools } \\
\hline & Camel & Cattle & Goat & Sheep & Total & $\mathbf{n}$ & $\%$ \\
\hline Amblyomma gemma & 1 & 11 & 4 & 1 & 17 & 1 & 5.9 \\
\hline Amblyomma hebraeum & 0 & 0 & 1 & 0 & 1 & 0 & 0.0 \\
\hline Amblyomma lepidum & 0 & 3 & 4 & 0 & 7 & 0 & 0.0 \\
\hline Amblyomma variegatum & 0 & 1 & 2 & 0 & 3 & 0 & 0.0 \\
\hline Amblyomma spp & 0 & 1 & 0 & 0 & 1 & 0 & 0.0 \\
\hline Rhipicephalus annulatus & 0 & 0 & 0 & 1 & 1 & 0 & 0.0 \\
\hline Hyalomma marginatum & 1 & 13 & 1 & 0 & 15 & 0 & 0.0 \\
\hline Hyalomma truncatum & 7 & 14 & 4 & 4 & 29 & 0 & 0.0 \\
\hline Hyalomma spp & 6 & 2 & 0 & 0 & 8 & 0 & 0.0 \\
\hline Rhipicephalus appendiculatus & 0 & 4 & 17 & 8 & 29 & 2 & 6.9 \\
\hline Rhipicephalus pulchellus & 15 & 79 & 79 & 28 & 201 & 8 & 4.0 \\
\hline Rhipicephalus evertsi evertsi & 0 & 25 & 19 & 24 & 68 & 10 & 14.7 \\
\hline Total & 30 & 153 & 131 & 66 & 380 & 21 & \\
\hline Coxiella PCR positive pools (n) & 0 & 7 & 7 & 7 & 21 & & \\
\hline Coxiella PCR positive pools (\%) & 0 & 4.6 & 5.3 & 10.6 & & & \\
\hline
\end{tabular}

\section{Data Availability}

The data used to support the findings of this study are available from the corresponding author upon request.

\section{Disclosure}

This material has been reviewed by the Walter Reed Army Institute of Research. There is no objection to its presentation and/or publication. The opinions or assertions contained herein are the private views of the author and are not to be construed as official or as reflecting true views of the Department of the Army or the Department of Defense. The investigators have adhered to the policies for protection of human subjects as prescribed in AR 70-25.

\section{Conflicts of Interest}

The authors declare no conflicts of interest.

\section{Acknowledgments}

This work was supported by the US Army Medical Research Directorate-Kenya, Arbovirus surveillance study. The authors thank the Arbovirus Incidence and Disease (AVID) program for providing part of the samples used in this study. They acknowledge Silvanos Opanda, Benjamin Opot, John Gachoya, James Mutisya, Francis Mulwa, Dunstone Beti, Philip Tunge, and Faith Sigei for their technical assistance and Santos Yalwala for generating the map. This work has been submitted with the permission of the Director, KEMRI.

\section{References}

[1] H. Sprong, E. Tijsse-Klasen, M. Langelaar et al., "Prevalence of coxiella burnetii in ticks after a large outbreak of Q Fever," Zoonoses and Public Health, vol. 59, no. 1, pp. 69-75, 2012.
[2] S. Vanderburg, M. P. Rubach, J. E. B. Halliday, S. Cleaveland, E. A. Reddy, and J. A. Crump, "Epidemiology of Coxiella burnetii infection in Africa: a OneHealth systematic review," PLOS Neglected Tropical Diseases, vol. 8, no. 4, Article ID e2787, 2014.

[3] O. G. Baca, "Pathogenesis of rickettsial infections emphasis on Q fever," European Journal of Epidemiology, vol. 7, no. 3, pp. 222228, 1991.

[4] S. N. Fard and M. Khalili, "PCR-detection of Coxiella burnetii in ticks collected from sheep and goats in Southeast Iran," Journal of Arthropod-Borne Diseases, vol. 5, no. 1, p. 1, 2011.

[5] M. Maurin and D. Raoult, "Q fever," Clinical Microbiology Reviews, vol. 12, no. 4, pp. 518-553, 1999.

[6] T. J. Marrie, "Q fever-A review," The Canadian Veterinary Journal, vol. 31, no. 8, pp. 555-563, 1990.

[7] L. Norlander, "Q fever epidemiology and pathogenesis," Microbes and Infection, vol. 2, no. 4, pp. 417-424, 2000.

[8] R. J. C. Hart, “The epidemiology of Q fever," Postgraduate Medical Journal, vol. 49, no. 574, pp. 535-538, 1973.

[9] S. J. Cutler, M. Bouzid, and R. R. Cutler, "Q fever," Journal of Infection, vol. 54, no. 4, pp. 313-318, 2007.

[10] Z. Woldehiwet, "Q fever (coxiellosis): Epidemiology and pathogenesis," Research in Veterinary Science, vol. 77, no. 2, pp. 93-100, 2004.

[11] N. A. Wardrop, L. F. Thomas, E. A. J. Cook et al., "The Sero-epidemiology of Coxiella burnetii in Humans and Cattle, Western Kenya: Evidence from a Cross-Sectional Study," PLOS Neglected Tropical Diseases, vol. 10, no. 10, Article ID e0005032, 2016.

[12] C. Eldin, C. Mélenotte, O. Mediannikov et al., "From Q fever to Coxiella burnetii infection: A paradigm change," Clinical Microbiology Reviews, vol. 30, no. 1, pp. 115-190, 2017.

[13] H. Honarmand, "Q fever: An old but still a poorly understood disease," Interdisciplinary Perspectives on Infectious Diseases, vol. $8,2012$.

[14] O. Duron, K. Sidi-Boumedine, E. Rousset, S. Moutailler, and E. Jourdain, "The Importance of Ticks in Q Fever Transmission: What Has (and Has Not) Been Demonstrated?" Trends in Parasitology, vol. 31, no. 11, pp. 536-552, 2015. 
[15] R. C. Pacheco, I. E. Echaide, R. N. Alves, M. E. Beletti, S. Nava, and M. B. Labruna, "Coxiella burnetii in ticks, argentina," Emerging Infectious Diseases, vol. 19, no. 2, pp. 344-346, 2013.

[16] T. Vilibic-Cavlek, J. Kucinar, S. Ljubin-Sternak et al., "Prevalence of Coxiella burnetii antibodies among febrile patients in Croatia, 2008-2010," Vector-Borne and Zoonotic Diseases, vol. 12, no. 4, pp. 293-296, 2012.

[17] E. Angelakis and D. Raoult, "Q fever," Veterinary Microbiology, vol. 140, no. 3-4, pp. 297-309, 2010.

[18] D. Ndeereh, G. Muchemi, A. Thaiyah, M. Otiende, S. AngeloneAlasaad, and M. J. Jowers, "Molecular survey of Coxiella burnetii in wildlife and ticks at wildlife-livestock interfaces in Kenya," Experimental and Applied Acarology, vol. 72, no. 3, pp. 277-289, 2017.

[19] M. Prabhu, W. L. Nicholson, A. J. Roche et al., "Q fever, spotted fever group, and typhus group rickettsioses among hospitalized febrile patients in Northern Tanzania," Clinical Infectious Diseases, vol. 53, no. 4, pp. e8-e15, 2011.

[20] B. H. Noden, F. I. Tshavuka, B. E. Van Der Colf, I. Chipare, and R. Wilkinson, "Exposure and risk factors to Coxiella burnetii, spotted fever group and typhus group rickettsiae, and Bartonella henselae among volunteer blood donors in Namibia," PLoS ONE, vol. 9, no. 9, Article ID e108674, 2014.

[21] E. Schelling, C. Diguimbaye, S. Daoud et al., "Brucellosis and Q-fever seroprevalences of nomadic pastoralists and their livestock in Chad," Preventive Veterinary Medicine, vol. 61, no. 4, pp. 279-293, 2003.

[22] E. Vanek and B. Thimm, "Q fever in Kenya. Serological investigations in man and domestic animals," East African Medical Journal, vol. 53, no. 12, pp. 678-684, 1976.

[23] J. Njeru, K. Henning, M. W. Pletz, R. Heller, and H. Neubauer, "Q fever is an old and neglected zoonotic disease in Kenya: A systematic review," BMC Public Health, vol. 16, no. 1, article no. 297, 2016.

[24] H. Koka, R. Sang, H. L. Kutima, and L. Musila, Detection of Spotted Fever Group Rickettsioses, Coxiella burnetii and Theileria orientalis in human blood and tick samples from pastoral communities in Kenya. Master of Science in Parasitology and Entomology [Master, thesis], Jomo Kenyatta University of Agriculture and Technology, Nairobi, Kenya, 2018.

[25] H. Koka, R. Sang, H. L. Kutima, and L. Musila, "The detection of spotted fever group rickettsia DNA in tick samples from pastoral communities in Kenya," Journal of Medical Entomology, vol. 54, no. 3, pp. 774-780, 2017.

[26] B. Kumsa, C. Socolovschi, L. Almeras, D. Raoult, and P. Parola, "Occurrence and genotyping of coxiella burnetii in ixodid ticks in oromia, Ethiopia," The American Journal of Tropical Medicine and Hygiene, vol. 93, no. 5, pp. 1074-1081, 2015.

[27] D. L. Knobel, A. N. Maina, S. J. Cutler et al., "Coxiella burnetii in humans, domestic ruminants, and ticks in rural Western Kenya," The American Journal of Tropical Medicine and Hygiene, vol. 88, no. 3, pp. 513-518, 2013.

[28] O. Mediannikov, F. Fenollar, C. Socolovschi et al., "Coxiella burnetii in humans and ticks in rural Senegal," PLOS Neglected Tropical Diseases, vol. 4, no. 4, 2010.

[29] A. L. Reye, O. G. Arinola, J. M. Hubschen, and C. P. Muller, "Pathogen prevalence in ticks collected from the vegetation and livestock in Nigeria," Applied and Environmental Microbiology, vol. 78, no. 8, pp. 2562-2568, 2012.

[30] A. R. Walker et al., Ticks of Domestic Animals in Africa: a Guide to Identification of Species, Bioscience Reports, Edinburgh Scotland, 2003.
[31] W. Depuy, V. Benka, A. Massey et al., "Q fever risk across a dynamic, heterogeneous landscape in Laikipia County, Kenya," EcoHealth, vol. 11, no. 3, pp. 429-433, 2014.

[32] R. D. Brown, “Q fever-veterinary aspects," East African Medical Journal, vol. 33, 1956.

[33] R. Kuley, E. Kuijt, M. A. Smits, H. I. J. Roest, H. E. Smith, and A. Bossers, "Genome plasticity and polymorphisms in critical genes correlate with increased virulence of Dutch outbreakrelated Coxiella burnetii strains," Frontiers in Microbiology, vol. $8,2017$.

[34] Zoonotic Disease Unit (ZDU), Q-Fever outbreak Investigation and Response, Baringo County, Kenya, 2014.

[35] O. W. Lwande, Z. Irura, C. Tigoi et al., "Seroprevalence of Crimean Congo hemorrhagic fever virus in Ijara District, Kenya," Vector-Borne and Zoonotic Diseases, vol. 12, no. 9, pp. 727-732, 2012.

[36] A. N. Maina, C. M. Farris, A. Odhiambo et al., "Q fever, scrub typhus, and rickettsial diseases in children, Kenya, 2011-2012," Emerging Infectious Diseases, vol. 22, no. 5, pp. 883-886, 2016. 


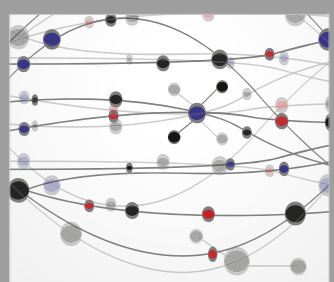

The Scientific World Journal
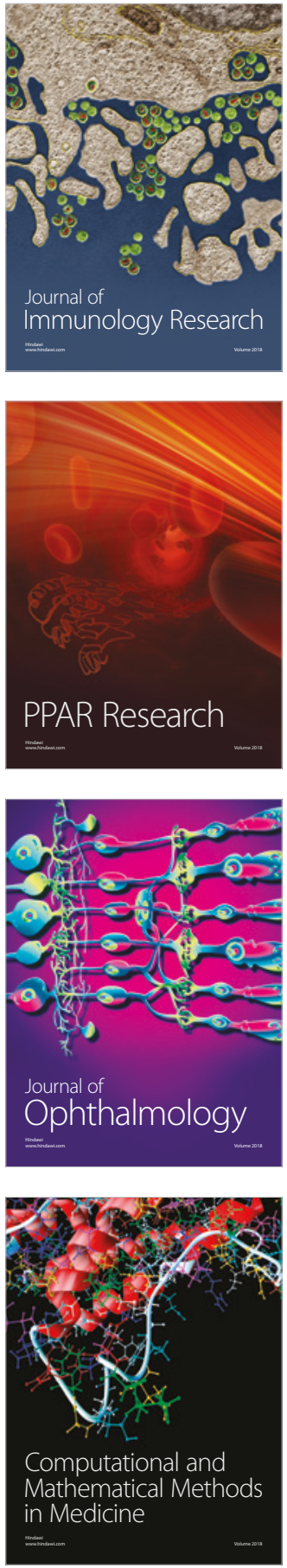

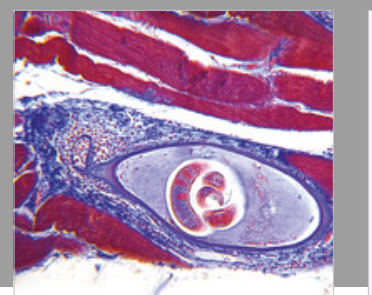

Gastroenterology Research and Practice

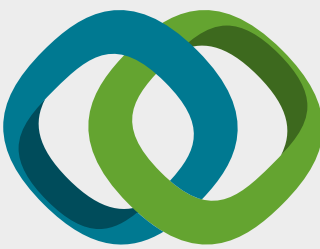

\section{Hindawi}

Submit your manuscripts at

www.hindawi.com
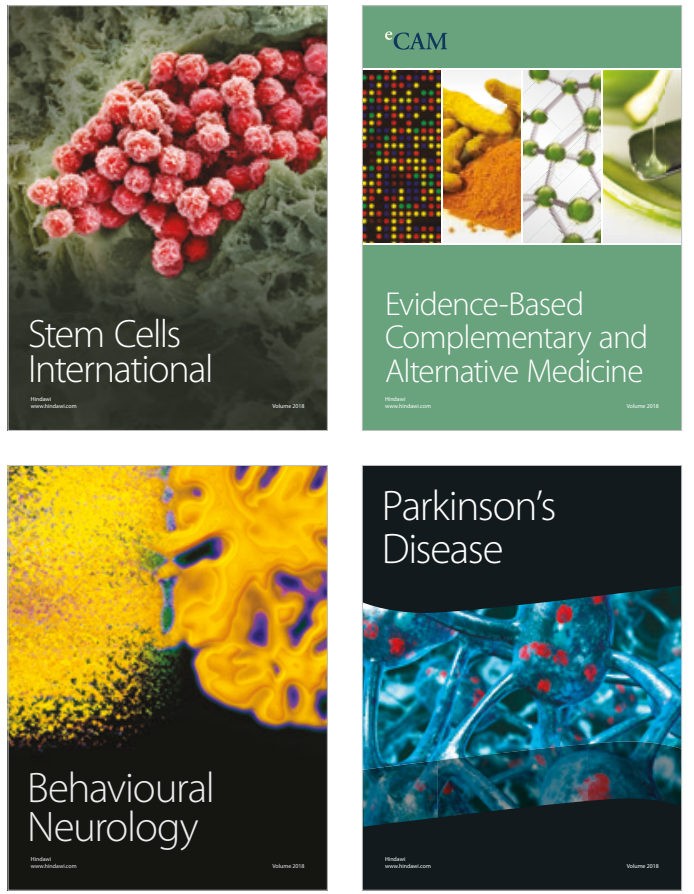

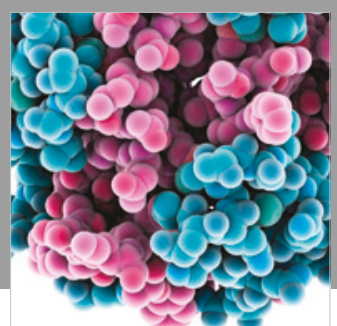

ournal of

Diabetes Research

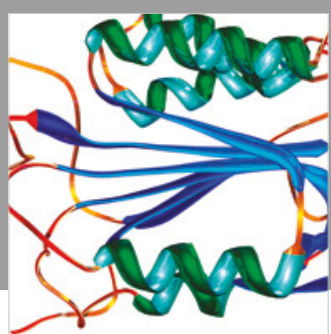

Disease Markers
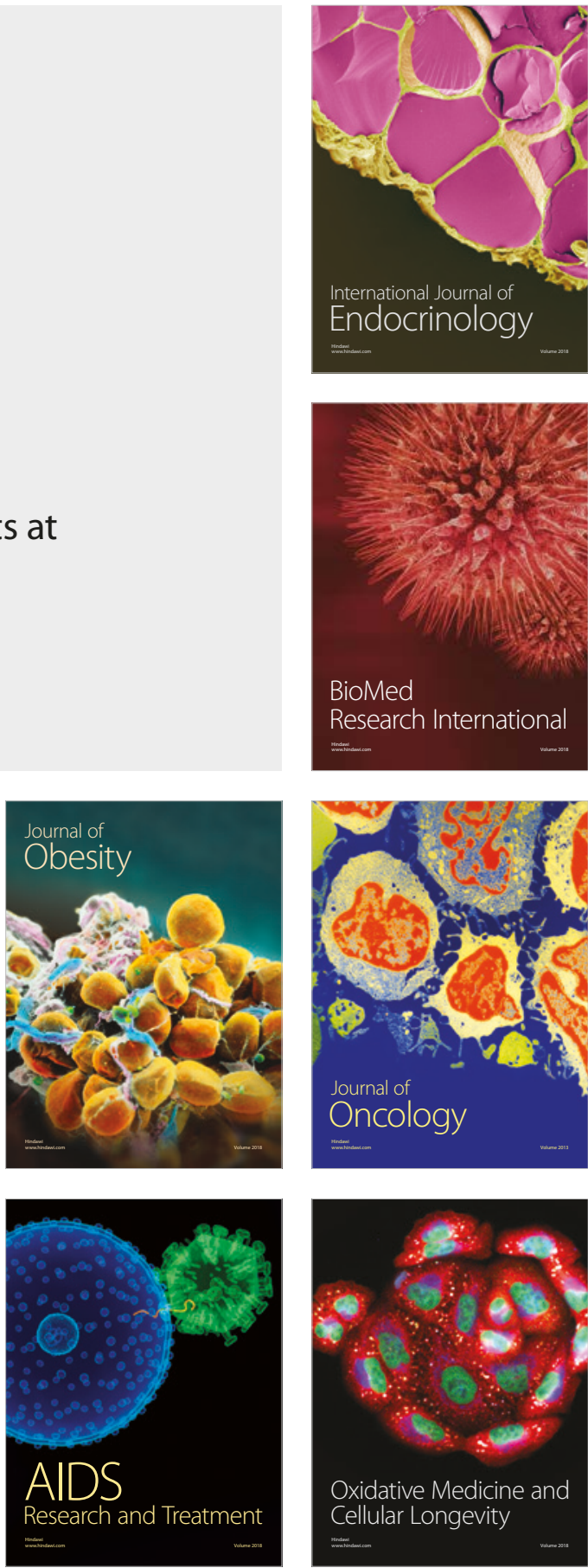